\title{
Survey of perceptions and practices among Canadian gastroenterologists regarding the prevention of venous thromboembolism for hospitalized inflammatory bowel disease patients
}

\author{
Roshan Razik MD ${ }^{1}$, Charles N Bernstein MD PhD FRCPC ${ }^{2}$, Justina Sam MD¹, \\ Reka Thanabalan BSc ${ }^{1}$, Geoffrey C Nguyen MD PhD FRCPC ${ }^{1}$
}

\begin{abstract}
R Razik, CN Bernstein, J Sam, R Thanabalan, GC Nguyen. Survey of perceptions and practices among Canadian gastroenterologists regarding the prevention of venous thromboembolism for hospitalized inflammatory bowel disease patients. Can J Gastroenterol 2012;26(11):795-798.
\end{abstract}

BACKGROUND: Patients with inflammatory bowel disease (IBD) who are hospitalized with disease flares are known to be at an increased risk of venous thromboembolism (VTE). This is a preventable complication; however, there is currently no standardized approach to the prevention and management of VTE.

OBJECTIVES: To characterize the opinions and general prophylaxis patterns of Canadian gastroenterologists and IBD experts.

METHODS: A survey questionnaire was sent to Canadian gastroenterologists affiliated with a medical school or IBD referral centre. Participants were required to be practicing physicians who had completed all of their training and had been involved in the care of IBD patients within the previous 12 months. Various clinical scenarios were presented and demographic data were solicited.

RESULTS: The majority of respondents were practicing in an academic setting (95\%) and considered themselves to be IBD experts or subspecialists $(71 \%)$. Eighty-three per cent reported providing VTE prophylaxis most, if not all of the time, and most (96\%) used pharmacological prophylaxis alone, usually heparin or one of its analogues. There was less consistency among respondents with respect to whether IBD patients in remission, but admitted for another condition, should be given prophylaxis. There was also less agreement regarding the duration of anticoagulation in patients with confirmed VTE.

CONCLUSION: There was a general consensus among academic gastroenterologists that IBD inpatients are at an increased risk for VTE and would benefit from VTE prophylaxis. However, areas of uncertainty still exist and the IBD community would benefit from evidencebased clinical practice guidelines to standardize the management of this important problem.

Key Words: Hospitalization; Inflammatory bowel disease; Prophylaxis; Venous thromboembolism

Tnflammatory bowel disease (IBD, comprised of ulcerative colitis and Crohn disease) has been shown to be associated with a variety of extraintestinal manifestations of disease. Venous thromboembolism (VTE) is an increasingly recognized feature of IBD $(1,2)$. Multiple studies have shown that IBD is an independent risk factor for thromboembolic complications because these patients incur a 1.5 - to 3.5fold higher risk of VTE compared with non-IBD patients (3-6).

A recent cohort study by Grainge et al (7) showed that the risk of VTE may be linked to the severity of inflammation, with patients in remission experiencing fewer VTE complications compared with those
Un sondage sur les perceptions et les pratiques chez les gastroentérologues canadiens au sujet de la prévention de la thromboembolie veineuse chez les patients hospitalisés à cause d'une maladie inflammatoire de l'intestin

HISTORIQUE : On sait que les patients atteints d'une maladie inflammatoire de l'intestin (MII) qui sont hospitalisés à cause d'une récidive sont plus vulnérables à une thromboembolie veineuse (TEV). Il s'agit d'une complication évitable, mais il n'existe pas de lignes directrices normalisées sur la prophylaxie de la TEV.

OBJECTIFS : Caractériser les opinions et les schèmes généraux de prophylaxie des gastroentérologues et des experts des MII du Canada. MÉTHODOLOGIE : Les chercheurs ont envoyé un sondage aux gastroentérologues canadiens affiliés à une faculté de médecine ou à un centre d'aiguillage des MII. Les participants devaient être des médecins en exercice qui avaient terminé leur formation et avaient soigné des patients atteints d'une MII au cours des 12 mois précédents. Divers scénarios cliniques leur étaient présentés, et ils devaient fournir des données démographiques.

RÉSULTATS : La majorité des répondants exerçaient dans un milieu universitaire (95\%) et se considéraient experts des MII ou surspécialistes (71\%). Quatre-vingt-trois pour cent ont déclaré prescrire une prophylaxie de la TEV la plupart du temps, sinon toujours, et la plupart (96\%) utilisaient seulement une prophylaxie pharmacologique, en général sous forme d'héparine ou de l'un de ses analogues. On constatait moins d'uniformité chez les répondants quant à l'intérêt d'administrer une prophylaxie aux patients atteints d'une MII en rémission, mais hospitalisés pour une autre maladie. On s'entendait également moins sur la durée de l'anticoagulothérapie chez les patients ayant une TEV confirmée.

CONCLUSION : Les gastroentérologues universitaires s'entendent généralement pour affirmer que les patients hospitalisés atteints d'une MII sont plus vulnérables à une TEV et profiteraient d'une prophylaxie de la TEV. Cependant, des incertitudes persistent, et le milieu des MII tirerait profit de lignes de pratique clinique probantes pour normaliser la prise en charge de ce problème important.

with active disease flares. Although ambulatory IBD patients were found to incur the most dramatic rise in relative risk, hospitalized IBD patients still showed the highest absolute risk $(3,7)$. Given the weight of evidence, many have recommended VTE prophylaxis for IBD patients hospitalized with disease flares. Methods of thromboprophylaxis can be mechanical, such as sequential compression devices, or pharmacological, such as subcutaneous heparin or its analogues.

Although VTE is a preventable complication, there are no standardized prophylactic practices in place to improve health outcomes for IBD inpatients. These patients tend to be younger, more mobile and present

${ }^{1}$ Mount Sinai Hospital Centre for Inflammatory Bowel Disease, University of Toronto, Toronto, Ontario; ${ }^{2}$ IBD Clinical and Research Centre, University

of Manitoba, Winnipeg, Manitoba

Correspondence: Dr Geoffrey C Nguyen, Mount Sinai Hospital Centre for Inflammatory Bowel Disease, 600 University Avenue, Suite 437, Toronto,

Ontario M5G 1X5. Telephone 416-586-4800 ext 2819, fax 416-586-5971, e-mail geoff.nguyen@utoronto.ca

Received for publication April 9, 2012. Accepted April 18, 2012 


\section{TABLE 1}

\section{Practice characteristics of respondents}

\begin{tabular}{lc}
\hline Years in practice, mean \pm SD & $14.1 \pm 10$ \\
Primary practice setting & \\
Academic & $54(95)$ \\
Community & $3(5)$ \\
Scope of practice & \\
Gastroenterology alone & $51(91)$ \\
Gastroenterology and general internal medicine & $4(7)$ \\
Gastrointestinal surgery & $0(0)$ \\
Other & $1(2)$ \\
Ever had IBD patient who developed VTE? & \\
Yes & $47(84)$ \\
No & $9(16)$ \\
If yes, did this VTE occur within the past 12 months? & \\
Yes & $23(50)$ \\
No & $23(50)$ \\
\hline
\end{tabular}

Data presented as $n(\%)$ unless otherwise indicated. IBD Inflammatory bowel disease; VTE Venous thromboembolism

with fewer comorbidities. As such, physicians may fail to recognize the importance of initiating VTE prophylaxis when these patients are hospitalized for disease flares. Additionally, given that IBD flares tend to present with some form of gastrointestinal bleeding, physicians may be further disinclined to administer prophylactic anticoagulation.

In the present study, we examined the opinions and general practices of Canadian gastroenterologists caring for hospitalized IBD patients. Specifically, we used survey data to portray a general picture of prophylaxis patterns and subsequent VTE management by gastroenterologists at academic and IBD referral centres. We also sought to identify factors that predicted the likelihood of prophylaxis among Canadian IBD experts.

\section{METHODS}

A questionnaire to gauge physician opinions regarding IBD patients admitted to hospital with disease flares was developed. A variety of clinical scenarios were presented and respondents were asked whether they would use anticoagulation for VTE prophylaxis in each of these patients, and why or why not. Demographic data were solicited. The questionnaire was administered via an online survey engine (Novi-Survey).

Canadian gastroenterologists who were affiliated with a Canadian medical school or IBD referral centre were invited to participate. E-mail and contact information were ascertained from departmental websites accessible in the public domain. Participants were approached via direct e-mail contact from the principal investigator (GC Nguyen).

After selecting the link to the survey site, participants were provided with a consent form. The respondents were offered the opportunity to electronically give consent or to decline the survey. Consent was implied if participants proceeded with the survey.

Invited physicians were prescreened before accessing the survey. Participants were required to be practicing physicians who had completed all of their training and had been involved in the care of IBD patients within the past 12 months.

A combination of mulitiple-choice questions, Likert scales and open text boxes were presented. All data were collected anonymously, and no responses were linked to respondents' e-mail addresses. Responses were coded with a unique identifier. Continuous variables were presented as means and SDs. Comparisons of categorical variables between subgroups were performed using the $\chi^{2}$ test or Fisher's exact test.

The study protocol was approved by the Research Ethics Board at Mount Sinai Hospital, Toronto, Ontario.

\section{RESULTS}

In total, 133 Canadian gastroenterologists affiliated with a university or academic teaching hospital were invited to participate. Overall, there were 63 respondents to the survey ( $47 \%$ response rate) and, among these, 56 met the inclusion criteria and completed the survey. The average length of time in independent practice ranged from one to 38 years (mean 14.1 years). The vast majority of participants reported their primary practice setting as academic in nature, with only $5.3 \%$ having a community-based practice (Table 1).

Most participants (91\%) had a practice primarily focused on gastroenterology, with only $7 \%$ reporting a mix of general internal medicine and gastroenterology. Nearly three-quarters of respondents (71\%) were self-reported subspecialists with IBD-specific expertise and $57 \%$ reported that at least $25 \%$ of their gastroenterology practice comprised IBD patients.

Approximately $16 \%$ of respondents reported that they have never had an IBD patient in their practice develop a VTE event (Table 1). Of those who did report an event, one-half stated that the event occurred within the past 12 months. All but one physician stated that they provide IBD care in the inpatient setting. Overall, 55\% reported that standard protocols or order sets for VTE prophylaxis existed in the hospital where they admited IBD patients, but slightly less than onehalf $(49 \%)$ stated that they were aware of published guidelines for VTE prophylaxis in hospitalized IBD patients.

Slightly more than one-half of respondents reported routinely assessing hospitalized IBD patients for signs or symptoms of VTE, while $46 \%$ stated that this was not part of their routine admission protocol. The majority of respondents $(83 \%)$ stated that they administered some form of VTE prophylaxis most, if not all of the time, for admitted patients (Figure 1A). Of those who routinely administered prophylactic interventions, almost all (96\%) used pharmacological prophylaxis alone. Indeed, Figure 1B illustrates that the most common form of pharmacological prophylaxis was heparin or one of its analogues (enoxaparin, dalteparin, tinzaparin). Overall, 64\% believed that VTE prophylaxis should be avoided only in patients with active, hemodynamically significant rectal bleeding but should be administered only once bleeding stops.

There was general agreement among respondents that hospitalized IBD patients, even those $<40$ years of age, were at an increased risk of VTE and that the presence of gastrointestinal bleeding in these patients was not a contraindication to pharmacological VTE prophylaxis. More than one-quarter (29\%) of respondents were either unsure or did not believe that VTE prophylaxis was necessary in hospitalized IBD patients who were mobile or were in clinical remission (hospitalized for another indication). There was also moderate uncertainty regarding the proven effectiveness of VTE prophylaxis, specifically in the IBD population, although the vast majority agreed that it was safe (Table 2).

Finally, Figure 2A presents the overall duration of treatment (in this case, pharmacological anticoagulation) endorsed by physicians if an IBD patient was diagnosed with his or her first episode of unprovoked VTE. Most respondents (83\%) agreed that they would refer such patients to a hematologist or thrombosis clinic for both initial management and long-term follow-up (Figure 2B).

There were no differences in the implementation of VTE prophylaxis between respondents who: reported themselves as IBD subspecialist and those who did not ( $81 \%$ versus $83 \%[\mathrm{P}=1.0])$; were and were not aware of IBD-related guidelines for VTE prophylaxis (78\% versus $89 \%[\mathrm{P}=0.5]$ ); admitted to hospitals with and without standardized VTE prophylaxis protocols ( $83 \%$ versus $83 \%[\mathrm{P}=1.0]$ ); and did and did not have previous experience with IBD patients with VTE (87\% versus $57 \%[\mathrm{P}=0.1])$.

\section{DISCUSSION}

The present survey depicts the current practices and attitudes toward the prevention and management of VTE in the IBD population among predominantly academic Canadian gastroenterologists. There is a strong body of epidemiological evidence to support the association between VTE and IBD. Additionally, VTE is considered to be among the leading causes of preventable in-hospital mortality.

In our survey, there was general agreement that hospitalized IBD patients are at an increased risk of VTE and should receive pharmacological 


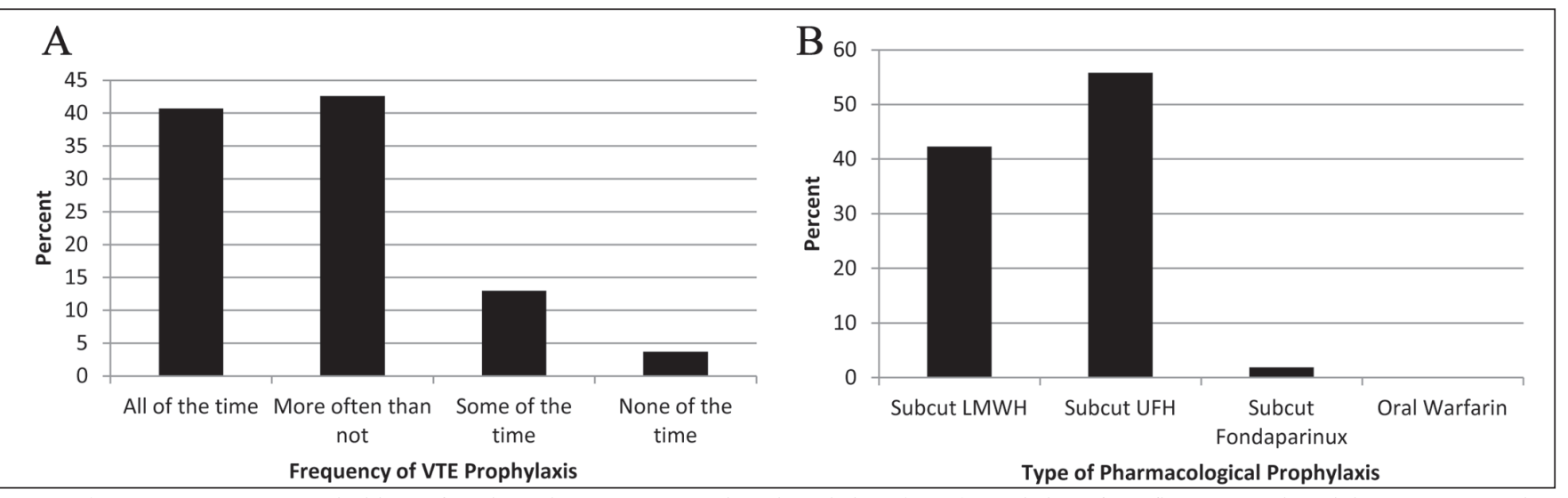

Figure 1) A Participants were asked how often they administer venous thromboembolism (VTE) prophylaxis for inflammatrory bowel disease patients when they are hospitalized. B First-line agents used for pharmacological VTE prophylaxis. LMWH Low-molecular weight heparin; Subcut Subcutaneous; UFH Unfractionated heparin

TABLE 2

Perceptions of indications, efficacy and safety of venous thromboembolism (VTE) prophylaxis among inflammatory bowel disease (IBD) inpatients

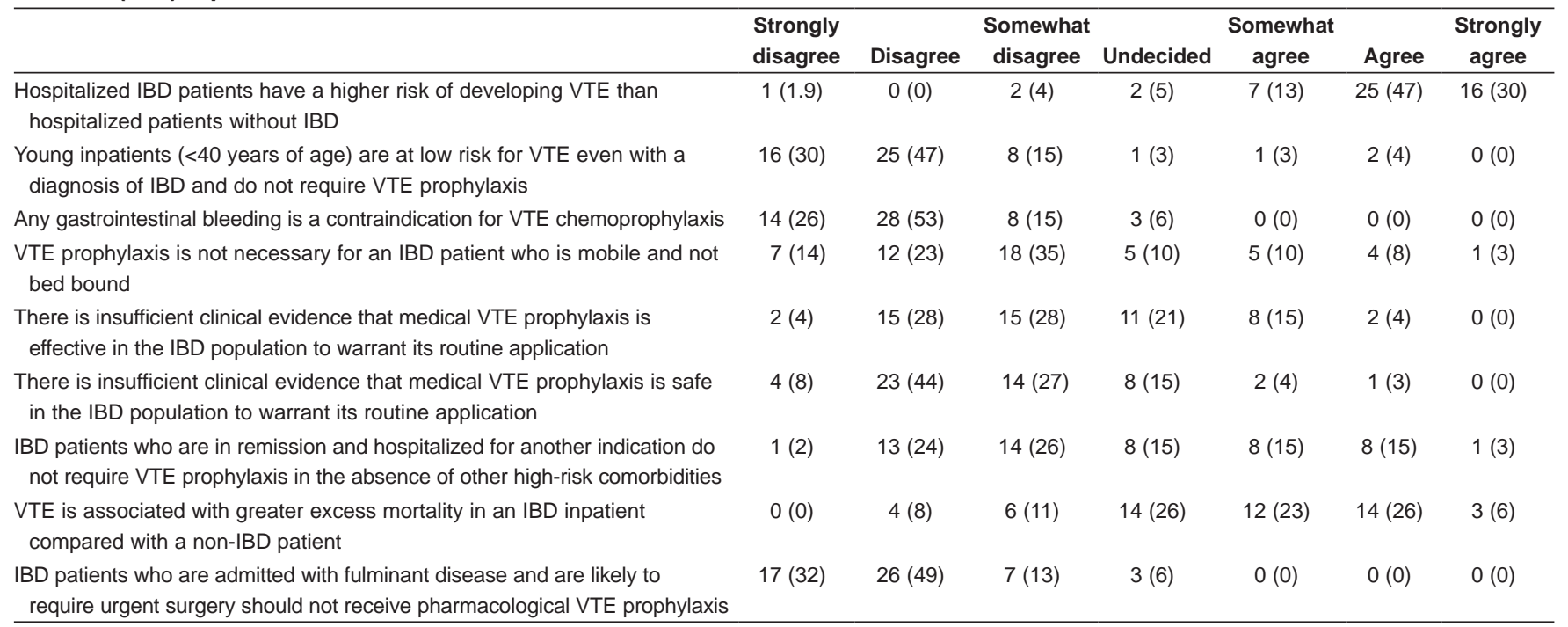
Data presented as $n$ (\%)

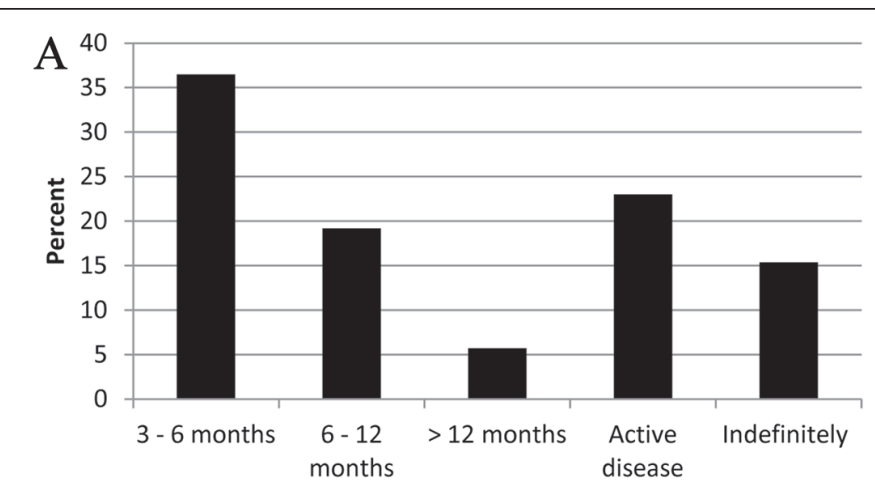

Duration of anticoagulation

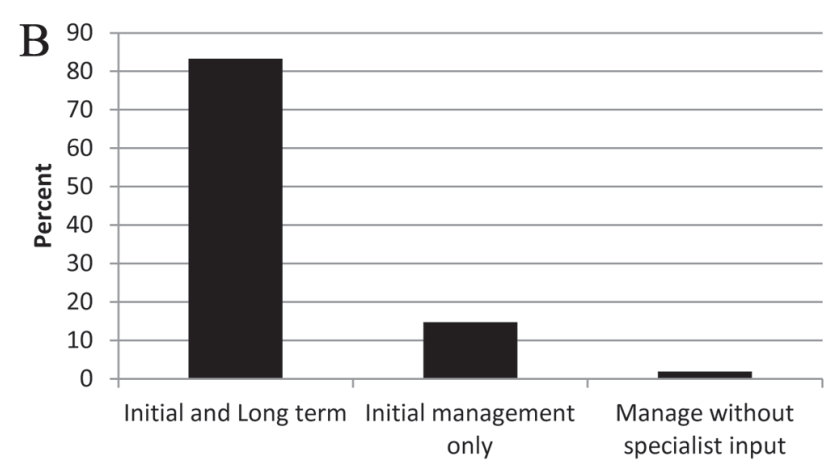

Referral to a hematologist

Figure 2) A Duration of treatment for an inflammatory bowel disease patient diagnosed with his or her first unprovoked venous thromboembolism. B Referral practices if an inflammatory bowel disease patient was diagnosed with venous thromboembolism

prophylaxis under most circumstances. In fact, more than $80 \%$ of respondents reported providing their hospitalized IBD patients with VTE prophylaxis most of the time. There is a substantial body of observational data that supports this practice $(2,4,7)$. There was also consensus among specialist respondents that pharmacological VTE prophylaxis is safe in active IBD and is not contraindicated in an IBD patient with active gastrointestinal bleeding unless there is hemodynamic comprise. Given the ubiquity of bleeding in active IBD, it is important to convey that most gastroenterologists favour VTE prophylaxis in this circumstance, especially among nonspecialists who may not have as much experience in caring for hospitalized IBD patients. The safety of pharmacological VTE prophylaxis is supported by a meta-analysis of 
eight clinical trials (8) that found no increased risk of major bleeding complications among patients who received heparin and low-molecular weight heparin for the primary treatment of ulcerative colitis.

There was, however, moderate uncertainty among the respondents as to whether it was necessary to provide VTE prophylaxis for IBD patients who were in remission and hospitalized for other indications or those who remained mobile during hospitalization. While several retrospective studies suggest that disease activity increases the risk of VTE, at least one large population-based study (7) suggested that even hospitalized IBD patients in clinical remission have a 1.7 -fold higher risk of VTE than the general population. In fact, the absolute risk of VTE for an inpatient with quiescent IBD is more than threefold higher than that of an ambulatory IBD patient in the midst of an active flare (20.9 per 1000 versus 6.4 per $1000 ; P=0.0001$ ). These data provide a rationale for offering VTE prophylaxis to hospitalized IBD patients in remission. In its consensus guidelines for VTE prophylaxis, the American College of Chest Physicians recommended pharmacological VTE prophylaxis for IBD patients who are confined to bed (9). The risk of VTE among IBD patients who are mobile during hospitalization is not well characterized. Furthermore, because mobility during hospitalization can be vacillating and subjective in nature, it could be argued that immobility should not be a criterion for VTE prophylaxis. However, as reflected by our survey results, whether VTE prophylaxis should be administered in IBD patients who are not immobile is somewhat controversial.

Another area of uncertainty uncovered by our survey was the optimal duration of anticoagulation once VTE is diagnosed. Our respondents recommended duration of therapy that ranged widely from three months to indefinitely, with nearly one-quarter stating they would continue therapy as long as active disease persisted. A multicentre study from Europe (5) suggested that approximately one-third of IBD patients with a first episode of VTE will experience a recurrent event within five years, which is a 2.5 -fold higher risk than that in non-IBD individuals with a first VTE. Although gastroenterologists had poor agreement on the duration of therapy, the vast majority reported they would refer to a hematologist for long-term management of VTE.

Our survey underscored several areas in which the prevention and management of VTE could be optimized. Slightly more than one-half of respondents reported having any standardized protocols for VTE prophylaxis in the hospitals where they admitted IBD patients. Standardized VTE risk assessment and protocols have been shown to increase the appropriate use of pharmacological VTE prophylaxis and should be implemented at most hospitals (10). Furthermore, fewer than one-half of respondents were aware of any guidelines for VTE prophylaxis that were relevant to IBD patients. The primary consensus statement that supports VTE prophylaxis in IBD patients comes from the American College of Chest Physicians, in which IBD is listed among many medical conditions that would warrant VTE prophylaxis if the patients were immobile (9). Other consensus guidelines from the British Society of Gastroenterology, the Canadian Association of Gastroenterology and the American College of Gastroenterology advocate VTE prophylaxis for patients admitted with severe ulcerative colitis (11-13). One of the principal reasons that IBD health providers may not be aware of these guidelines is that their target audience is not North American gastroenterologists. This deficiency underscores the need for IBD-specific consensus guidelines that address VTE prophylaxis in a Canadian context.

Among the limitations of our survey was that our responses were elicited from predominantly academic specialists and may not be generalizable to all gastroenterologists, particularly those practicing in the community. This deficiency may be compounded by survey response bias in which those who responded to the survey may have had particular interest or experience with VTE in the IBD population. Thus, the high rate of reported VTE prophylaxis in the present study may overestimate true rates among all gastroenterologists in Canada. Furthermore, self-reported rates of VTE may inherently overestimate actual practices. The Venous thromboembolism risk and prophylaxis in the acute hospital care setting: a multinational cross-sectional study (ENDORSE) study demonstrated that only $40 \%$ of acutely ill hospitalized patients received VTE prophylaxis in accordance with the American College of Chest Physicians guidelines (14). This discrepancy highlights the need to conduct multicentre audits of actual VTE prophylaxis rates among hospitalized IBD patients.

\section{SUMMARY}

Our survey demonstrated that there is general agreement among academic gastroenterologists in Canada that IBD patients are at an increased risk of VTE and its associated morbidity and mortality, and should receive VTE prophylaxis under most circumstances. We should strive to build on this level of agreement and evidencebased data to formally develop Canadian-based position statements or guidelines that will increase awareness of VTE in the vulnerable IBD population and optimize its prevention during hospitalization. There are areas of uncertainty, such as duration of anticoagulation for diagnosed VTE, that would benefit from a multidisciplinary working group including experts in the field of IBD and hematology charged with the task of developing a standardized and algorithmic approach to treating VTE in IBD patients.

DISCLOSURES: The authors have no financial disclosures or conflicts of interest to declare.

\section{REFERENCES}

1. Miehsler W, Reinisch W, Valic E, et al. Is inflammatory bowel disease an independent and disease-specific risk factor for thromboembolism? Gut 2004;53:542-8.

2. Nguyen GC, Sam J. Rising prevalence of venous thromboembolism and its impact on mortality among hospitalized inflammatory bowel disease patients. Am J Gastroenterol 2008;103:2272-80.

3. Murthy SK, Nguyen GC. Venous thromboembolism in inflammatory bowel disease: An epidemiological review. Am J Gastroenterol 2011;106:713-18.

4. Bernstein CN, Blanchard JF, Houston DS, Wajda A. The incidence of deep venous thrombosis and pulmonary embolism among patients with inflammatory bowel disease: A population-based cohort study. Thromb Haemost 2004;85:430-4.

5. Novacek G, Weltermann A, Sobala A, et al. Inflammatory bowel disease is a risk factor for recurrent venous thromboembolism. Gastroenterology 2010;139:779-87.

6. Koutroubakis IE. Which patients with IBD are at risk of venous thromboembolism? Nat Rev Gastroenterol Hepatol 2010;7:307-8.

7. Grainge MJ, West J, Card TR. Venous thromboembolism during active disease and remission in inflammatory bowel disease: A cohort study. Lancet 2010;375:657-63.

8. Shen J, Ran ZH, Tong JL, Xiao SD. Meta-analysis: The utility and safety of heparin in the treatment of active ulcerative colitis. Aliment Pharmacol Ther 2007;26:653-63.

9. Geerts W II, Bergqvist D, Pineo GF, et al. Prevention of venous thromboembolism: American College of Chest Physicians Evidence-Based Clinical Practice Guidelines, 8th edn. Chest 2008;133(Suppl 6):381S-453S.

10. Maynard G, Stein J. Designing and implementing effective venous thromboembolism prevention protocols: Lessons from collaborative efforts. J Thromb Thrombolysis 2010;29:159-66.

11. Mowat C, Cole A, Windsor A, et al. Guidelines for the management of inflammatory bowel disease in adults. Gut 2011;60:571-607.

12. Kornbluth A, Sachar DB. Ulcerative colitis practice guidelines in adults: American College of Gastroenterology. Practice Parameters Committee. Am J Gastroenterol 2010;105:501-23.

13. Bitton A, Buie D, Enns R, et al. Treatment of hospitalized adult patients with severe ulcerative colitis: Toronto consensus statements. Am J Gastroenterol 2012;107:179-94.

14. Cohen AT, TapsonVF, Bergmann JF, et al. Venous thromboembolism risk and prophylaxis in the acute hospital care setting (ENDORSE study): A multinational cross-sectional study. Lancet 2008;371:387-94. 


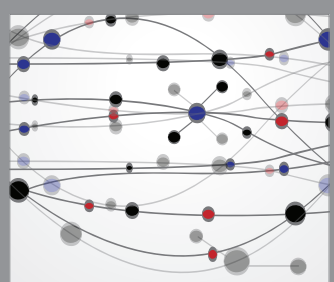

The Scientific World Journal
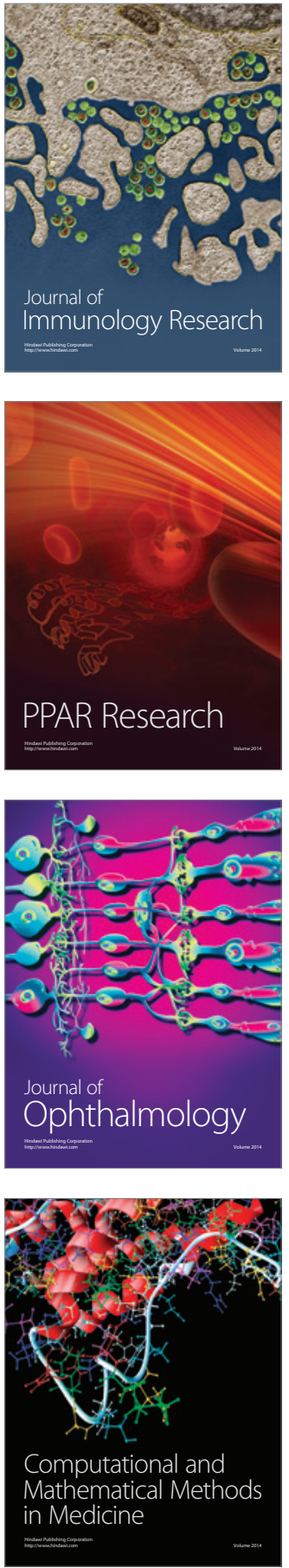

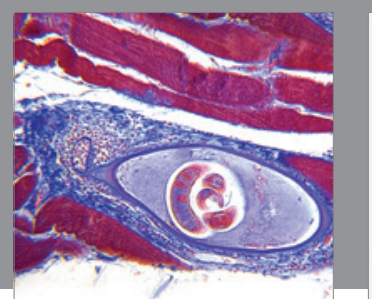

Gastroenterology Research and Practice

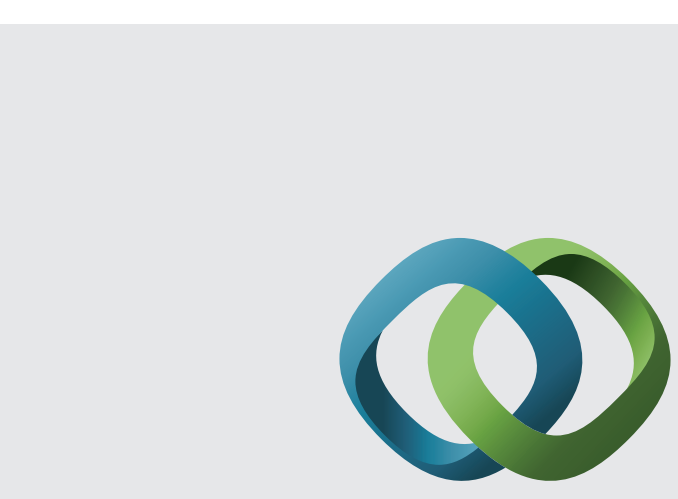

\section{Hindawi}

Submit your manuscripts at

http://www.hindawi.com
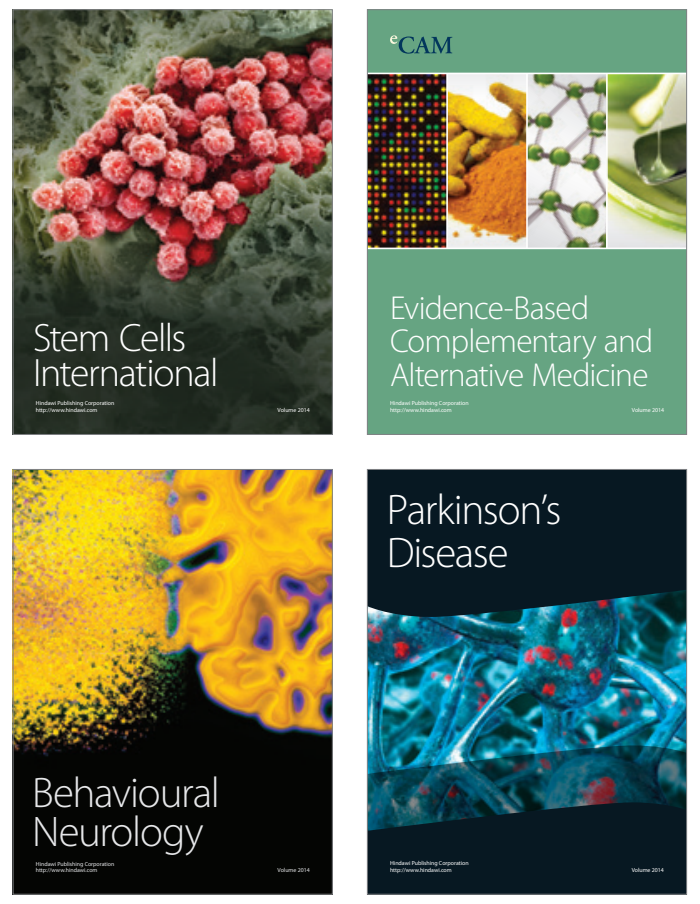
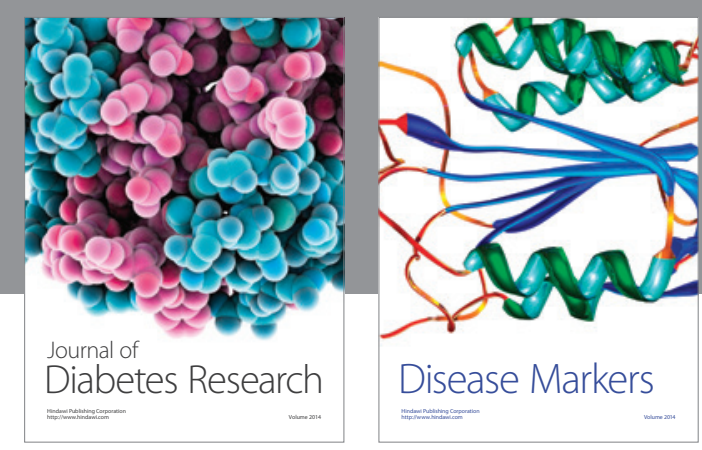

Disease Markers
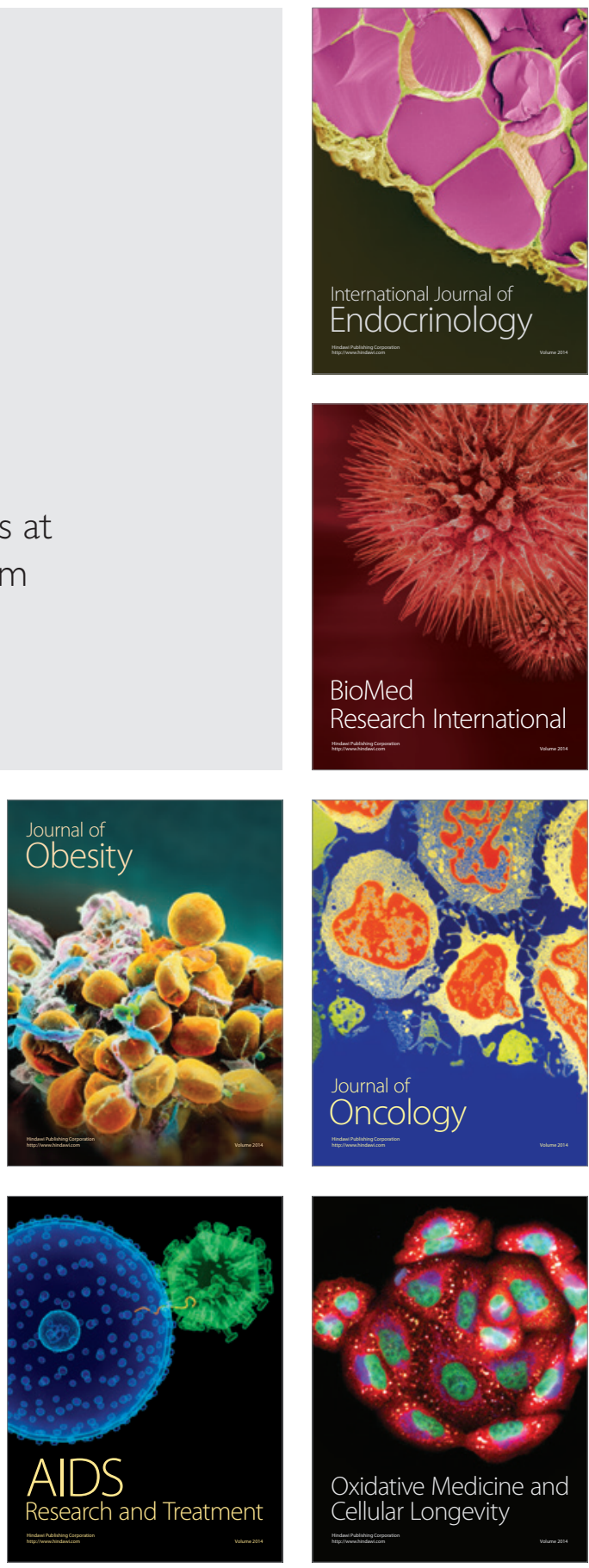